\title{
SYSTEMIC BUILDING LCA
}

\author{
Miroslaw Dytczak \\ AGH University of Science and Technology \\ Kraków, Poland \\ E-mail:mdytczak@gmail.com,mdytczak@zarz.agh.edu.pl \\ Grzegorz Ginda \\ AGH University of Science and Technology \\ Kraków, Poland \\ E-mail: gg.ginda@gmail.com,gginda@zarz.agh.edu.pl
}

\begin{abstract}
Life Cycle Assessment (LCA) is a universal framework for systemic evaluation of products influence on natural environment. It has been also extended to cover different specialized areas e.g. buildings. Buildings are specific products of construction processes. They are meant to provide diverse services to their users and must conform to different requirements. They comprise complex technical systems, therefore. The provided services and established requirements often deal with contradictory goals. Note, that some features of the buildings, as well as, the interrelations between components of the buildings, and between the buildings and natural environment may be intangible. Feedback may also appear. It is difficult, therefore, to evaluate performance of buildings credibly. The modifications of the core LCA approach are proposed, therefore, in the paper. They are intended to support the reliable application of LCA methodology in the case of buildings. The proposed modifications apply ANP and DEMATEL to cope with possible intangibility and feedback. The utilization of these two universal approaches allows to obtain reliable results for LCA-based building evaluation.
\end{abstract}

Keywords: LCA, building, influence, environment, overall evaluation, intangibility, feedback. 


\section{Introduction}

Buildings provide different services to their users. Building components have also to conform to diverse requirements and regulations to provide appropriate levels of safety and comfort for the users. These issues make buildings complex technical objects. One should not forget that buildings are products of construction processes. And, as such, can be evaluated as every other product by the means of a similar methodology. For example, LCA may be applied to evaluate the environmental impact of a building while considering its full life cycle - from gaining the necessary raw materials, through the production of building components, building erection and usage, to demolition and the recycling of building materials (Cabesa et al., 2014).

The complexity of the contemporary buildings results in the application of diverse components to erect them and to provide appropriate conditions for their successful performance. Note, that the components are devoted to different and often conflicting goals. There may also occur other problems which hinder reliable evaluation of building impact on environment. Firstly, the intangibility of building component features, as well as, the intangibility of relations among the components, and between the building and natural environment may occur. Secondly, feedback between building components, and between building and natural environment may also appear. Application of LCA needs appropriate support, therefore, to cope with such issues successfully.

It seems that the application of ANP can help in this regard. Note, however, that it depends on the information about directions of the relations between the components of applied network model. Such the information may be delivered by specialized methods. For example, DEMATEL (Fontela \& Gabus 1976) may be applied in this regard. The DEMATEL-ANP-based framework for LCA approach is presented, therefore, in the paper.

\section{Literature Review}

Let us begin from the outline of LCA methodology (Guinée, 2002). It results from the needs related to the contemporary world problems. The environmental pollution, the continuous increase in energy demands, the scarcity and exhausting of energy sources, the continuous increase in wastes volume are the main environmental concerns. The life cycle evaluation aims at including the influence of all factors, related to a considered product, which may influence natural environment. LCA application deals with the creation of a structure which describes all possible relations between the factors. The relations between input factors (material, energy) and output factors (generated wastes and their influence o natural environment) are dealt with carefully in this regard, while considering each life cycle phase for a product. Note, that the indirect influence of applied materials and energy on environment should is also addressed. Contemporary research trends consider LCA in the sustainability context. The context deals with environmental, economic and social dimensions (Finkbeiner, 2011).

There are two principal goals of LCA applications. The first goal deals with the evaluation of the overall influence of product life cycle on natural environment. Such approach to LCA enables to compare the environmental performance of different products or different design alternatives available for the same product. The other goal pertains to the identification of the key factors of the product influence on the natural environment. The results of the identification allow to concentrate on the most promising means for product improvement.

International Symposium of 2

Washington, D. C. the Analytic Hierarchy June 29 - July 2, 2014

Process 
A general step-wise procedure is utilized to implement LCA. The introductory analysis is devoted to the collecting of the preliminary data about the relations between different factors. The collected data are then applied to particuliarize and extend the relations. Additional identified information is also included in the following procedure steps. Comprehensive systemic approach to environmental influences makes LCA a viable alternative for the decision support while selecting the environmentally preferable products and while evaluating and optimizing construction processes. The curreant LCA research interests may be divided into 2 specific groups (Cabeza, et al. 2014). The first group deals with the building industry as a whole. The second pertains to the building sector and individual buildings. The selection of construction products, the evaluation of construction systems and process, as well as, the development in LCA methodology and decision support tools are dealt with in the case of the first group of research interests. The second group pertains to the individual problems related to peculiar construction industry products: residential and non-residental buildings, and specialized civil engineering structures.

There are specific kinds of LCA available for the assessment of buildings. They correspond with the considerable energy consumption and total costs related to the production of building components, building usage and dismantling, as well as, the recycling of building materials. Life Cycle Energy Analysis (LCEA) and Life Cycle Cost Analysis (LCCA) are applied in this regard. LCEA deals with the estimation of the overall demand for energy. LCCA allows to assess the overall cost of ownership. All costs resulting from building acquiring, owning, and disposing a building are included in this regard. The distinction of LCEA and LCCA is fully justified because the building industry influences the overall consumption of both natural resources and energy, as well as, overall hazardous emissions a lot. Note, that a building generates the demand for energy and wastes in both the direct way (during construction, erection, utilization and dismantling) and indirect way (production and recycling of building components).

It is often argued that the main LCA deficiency deals with a lack of the objectivity. The special attention is drawn, therefore, to introduce the improvements which deal with the evaluation of the influence of involved factors and weighting their individual importance. The application of the appropriate decision support tools becomes, thus, indispensable while implementing LCA in practice. Let us also observe, that LCA application should provide readable indices for the environmental impact measurement. It seems that ANP application is capable of helping a lot in this regard.

\section{Objectives}

The main objective of the paper is to provide intangibility and feedback-aware framework for LCA support. The framework should facilitate conducting the environmental impact analysis. It has also to deliver the synthetic index making reliable the comparison and evaluation of different construction industry products easier. The multi-dimensional nature of environmental impact should be included in this regard. The framework is also intended to provide the necessary means for coping with diverse LCA subjects and goals.

\section{Methodology}

Note, that the relations between factors related to the impact of a building on natural environment should be defined credibly to make the successful application of ANP 
possible. Therefore, DEMATEL is applied in the paper to provide the credible means for the identification of the ANP network. The proven usability of DEMATEL assures obtaining the proper results in this regard (Liou et al. 2009).

The direct information gathered from appointed experts is applied to deliver the necessary data for both DEMATEL and ANP application. Application of other - indirect sources of data, e.g. financial, economic, social, and environmental reports, is also possible. Both DEMATEL and ANP provide necessary means for the aggregation of data originated from different sources. For example, simple arithmetic average is applied in DEMATEL to combine the opinions provided by independent experts. Casual techniques - simple or geometric average - are applied to aggregate the opinions provided by the independent experts in the ANP case. All inconsistency problems are addressed in a usual way - by the means of RI and GCI. indices.

\section{Model Analysis}

An applied LCA-support framework is tailored to the specific needs of a problem at hand. It consist of 3 steps (Fig.1). The first step deals with DEMATEL application to identify the credible ANP network. Several steps may be needed to obtain the appropriate network. Each step improves a unique structure of relations between the factors, and between the factors and natural environment. The ANP model is formulated and applied in the second step to obtain data that are necessary to estimate environmental impact of a considered building. The third step provides the environmental index value.

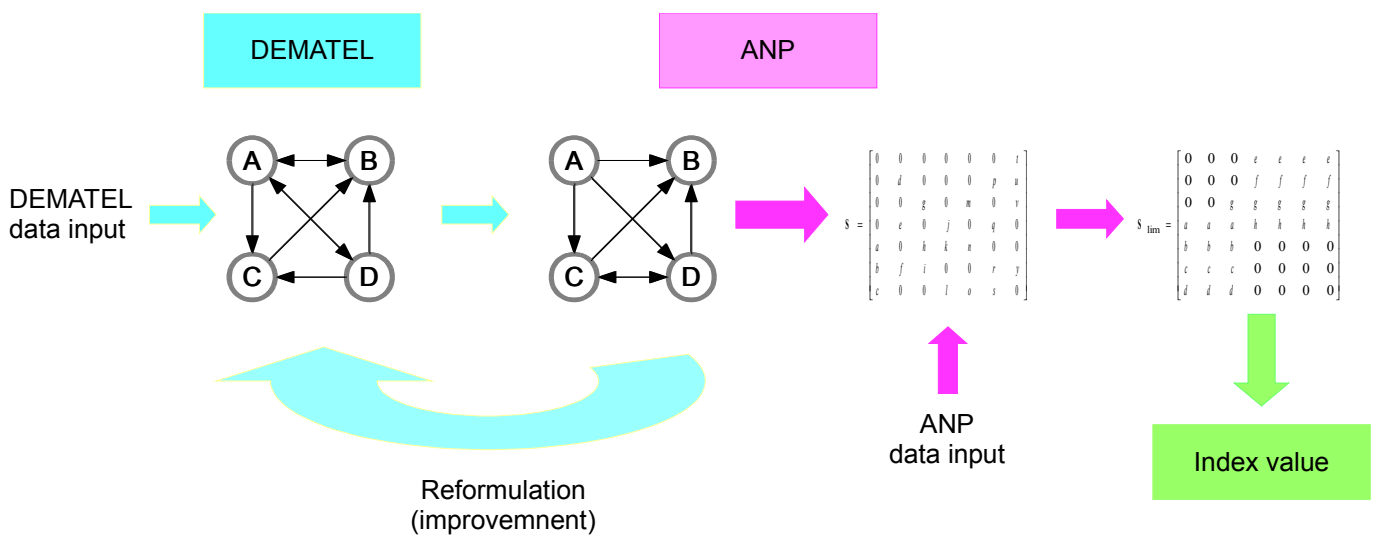

Fig.1. DEMATEL-ANP-based framework for the LCA support

\section{Limitations}

The presented framework for LCA support is still under construction. The main problem with the implementation of approach deals with the credibility of expert opinions. It seems that the proposed framework for LCA should be rather considered like a component of a larger decision support system. Such the system would provide the necessary means for both selecting appropriate experts and supporting them in preparing necessary data for LCA application.

Let us also observe, that the proven flexibility and other merits of DEMATEL and ANP make it possible to enhance the framework in various ways if needed.

International Symposium of 


\section{Conclusions}

DEMATEL and ANP complement each other while providing the necessary means for reliable coping with decision making problems that deal with the intangibility and feedback. Complex nature o buildings, building structures and construction industry results in LCA problems that deal with both the intangibility and feedback. The DEMATEL-ANP approach proves useful, therefore, for supporting LCA related to buildings. The flexibility and other advantages of DEMATEL and ANP facilitate exploiting their merits. Therefore, the presented approach seems to be a vital supplement for existing LCA tools. The actual benefits resulting from the application of the framework result from its application to the individual LCA cases, however.

\section{Key References}

Fontela E., Gabus A. (1976). The DEMATEL Observer. Geneva: Batelle Memorial Research Institute.

Guinée J. (Ed.) (2002). Handbook on Life Cycle Assessment. Springer.

Finkbeiner M. (Ed.) (2011). Towards Life Cycle Sustainability Management. Springer.

Luisa F. Cabeza, Lídia Rincón, Virginia Vilariño, Gabriel Pérez, Albert Castell. (2014). Life cycle assessment (LCA) and life cycle energy analysis (LCEA) of buildings and the building sector: A review. Renewable and Sustainable Energy Reviews, 29, 394-416.

Liou J.J.H., Yeh W.-C., Lo Y.-C., Lin C.-L. (2009). Developing a hybrid multi-criteria model for selection of outsurcing providers. Proceedings of the International Symposium on the Analytic Hierarchy Process ISAHP 2009, July 29 - August 1, 2009 Pittsburgh, PA. 